\title{
EFEITO ANTIBIOFILME DO EXTRATO DE ALCACHOFRA (Cynara scolymus) VERIFICADO SOBRE Pseudomonas aeruginosa
}

\author{
Jonatas Rafael de Oliveira ${ }^{1}$ \\ Leandro Wagner Figueira ${ }^{2}$ \\ Fábia Lugli Sper ${ }^{3}$ \\ Vanessa Marques Meccatti ${ }^{4}$ \\ Letícia de Oliveira $^{5}$ \\ Luciane Dias de Oliveira ${ }^{6}$
}

Resumo: Alcachofra é um vegetal utilizado na culinária e algumas de suas ações biológicas foram descritas, como ação antioxidante e anti-inflamatória, porém foi pouco relatada sua atividade sobre microorganismos. Com isso, o objetivo do presente estudo foi verificar o efeito antimicrobiano do extrato de alcachofra sobre $P$. aeruginosa. Primeiramente, foi verificada a ação deste extrato sobre cultura planctônica, pelo método de microdiluição em caldo (50 a 0,09 mg/mL), em seguida, sobre biofilme formado por $48 \mathrm{~h}$ em poços de placas de microtitulação, com exposição de $5 \mathrm{~min}$ ao extrato de alcachofra (200 $\mathrm{mg} / \mathrm{mL})$. Solução salina 0,9\% e clorexidina 0,12\% foram utilizadas como controles. As reduções do biofilme foram verificadas pelo teste de MTT e analisadas estatisticamente por ANOVA e Tukey Test $(p \leq 0,05)$. Em culturas planctônicas foi verificado que as concentrações avaliadas não foram efetivas (CIM>50 mg/mL), contudo, sobre o biofilme foi verificada redução significativa de $42 \pm 16 \%$. Desta forma, pode-se concluir que o extrato de alcachofra apresentou efeito antimicrobiano sobre biofilme de $P$. aeruginosa.

Palavras-chave: Alcachofra; Cynara scolymus; Biofilme; Pseudomonas aeruginosa.

\footnotetext{
1 Pós-graduação em Biopatologia Bucal/Instituto de Ciência e Tecnologia - UNESP, Departamento de Biociências e Diagnóstico Bucal, Brasil. E-mail: jroliveira16@hotmail.com.

2 Pós-graduação em Biopatologia Bucal/Instituto de Ciência e Tecnologia - UNESP, Departamento de Biociências e Diagnóstico Bucal, Brasil. E-mail: leandrowf@live.com.

3 Pós-graduação em Biopatologia Bucal/Instituto de Ciência e Tecnologia - UNESP, Departamento de Biociências e Diagnóstico Bucal, Brasil. E-mail: fabiafarma@hotmail.com.

${ }^{4}$ Pós-graduação em Biopatologia Bucal/Instituto de Ciência e Tecnologia - UNESP, Departamento de Biociências e Diagnóstico Bucal, Brasil. E-mail: vanessa.tep@hotmail.com.

5 Pós-graduação em Biopatologia Bucal/Instituto de Ciência e Tecnologia - UNESP, Departamento de Biociências e Diagnóstico Bucal, Brasil. E-mail: leticia.4541@gmail.com.

${ }^{6}$ Pós-graduação em Biopatologia Bucal/Instituto de Ciência e Tecnologia - UNESP, Departamento de Biociências e Diagnóstico Bucal, Brasil. E-mail: luciane@ict.unesp.br.
} 